\title{
Can We Change Autoimmunity in Type 1 Diabetes through Insulin Injection or Oral Insulin?
}

\section{Muyun Cao}

\author{
Department of Biomedical Engineering, University of British Columbia, Vancouver, Canada \\ Address for correspondence: Muyun Cao, University of British Columbia, Vancouver, Canada. \\ E-mail: muyuncaomc@gmail.com
}

\section{Abstract}

This article reviews recent immunotherapy studies of Type 1 diabetes (T1D) which is an autoimmune disease. Researchers show that injecting human proinsulin peptides can safely modulate the immune system and affect beta-cell function in T1D, but oral insulin consumption does not reduce the onset of T1D in individuals at the early stage of the disease.

Keywords: Autoimmunity, Insulin, Immunotherapy, Type 1 diabetes

\section{Introduction}

The incidence of Type 1 diabetes (T1D) is increasing worldwide. The complications resulting from high blood glucose are unavoidable and cause huge burdens on patients and the public health-care system. The need to develop therapies to control and delay T1D progression is urgent. Two recent clinical studies on insulin immunotherapies have shown to modulate autoimmunity and restore immune tolerance. Ali et al. given intradermal injections of human proinsulin peptides to new T1D patients. The treatment is safe, has immune modulation effects, and does not accelerate betacell function loss. ${ }^{[1]}$ Greenbaum et al. reported that oral insulin administration cannot delay or prevent T1D in autoantibody-positive subjects. ${ }^{[2]}$

T1D is an autoimmune disorder involving the immune system attacking the islet beta-cells. The autoreactive effector CD4 T-cells releases pro-inflammatory cytokines. The $\mathrm{T}_{\text {reg }}$ cells are compromised, and the regulatory autoreactive CD4 T-cells release deficient interleukin-10 (IL-10) to suppress the effector T-cells. ${ }^{[3]}$ The previous antigen-specific immunotherapies (ASIs) have shown some benefits, but balanced efficacy and safety have not been achieved. Therapeutic vaccines have resulted in T1D remission by deleting effector T-cells and stimulating the regulatory effects of T-cells, but clinical outcomes have not been reported. ${ }^{[4]}$ High-dose oral insulin has shown protective immunological effects in a few children at high T1D risk but also has shown adverse effects. ${ }^{[5]}$

\section{Insulin Injection Immunotherapy}

Ali et al. conducted a randomized, double-blind, placebo-controlled peptide immunotherapy (PIT) study. T1D subjects receive intradermal injections according to their randomized groups, as shown in Figure 1. The researchers use proinsulin peptide to represent the epitopes of T1D-related autoantigens. They monitor the subjects for safety, glycemic control, autoantibodies, insulin usage, and glycated hemoglobin (HbA1c). They use insulin dose-adjusted HbA1c (IDAA1c) to evaluate the combined impact of insulin usage and $\mathrm{HbA} 1 \mathrm{c}$.

Ali et al. reported that the injected peptide is well tolerated without severe adverse events or hypersensitivity. The placebo group shows significantly decreased C-peptide, increasing insulin usage, increasing $\mathrm{HbA} 1 \mathrm{c}$, and increasing IDAA1c compared with the baselines over 12 months. In contrast, the treatment group shows stable C-peptide levels (only showing a significant loss after 12 months in the high-frequency group), stable average insulin usages, and declined or stable HbA1c compared with the baselines or the placebo group. The treatment group also shows stable IDAA1c compared with the baselines but lower IDAA1c compared with the placebo group. Moreover, researchers further characterize immune changes. They classify subjects

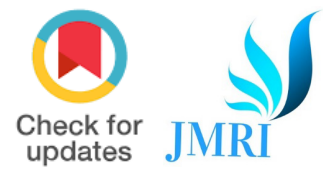

How to cite this article: Cao M. Can We Change Autoimmunity in Type 1 Diabetes through Insulin Injection or Oral Insulin? J Med Res Innov. 2020;4(1):e000196.

Doi: 10.32892/jmri.196

Publication history: Received: 07-10-2019 Accepted: 09-11-2019 Published: 09-11-2019

Editor: Dr. Varshil Mehta

Copyright: Cao M. This is an open-access article distributed under the terms of the Creative Commons Attribution License CC-BY 4.0., which permits unrestricted use, distribution, and reproduction in any medium, provided the original author and sources are credited.

Funding: NIL

Conflict of Interest: NIL

MK Medkrux 


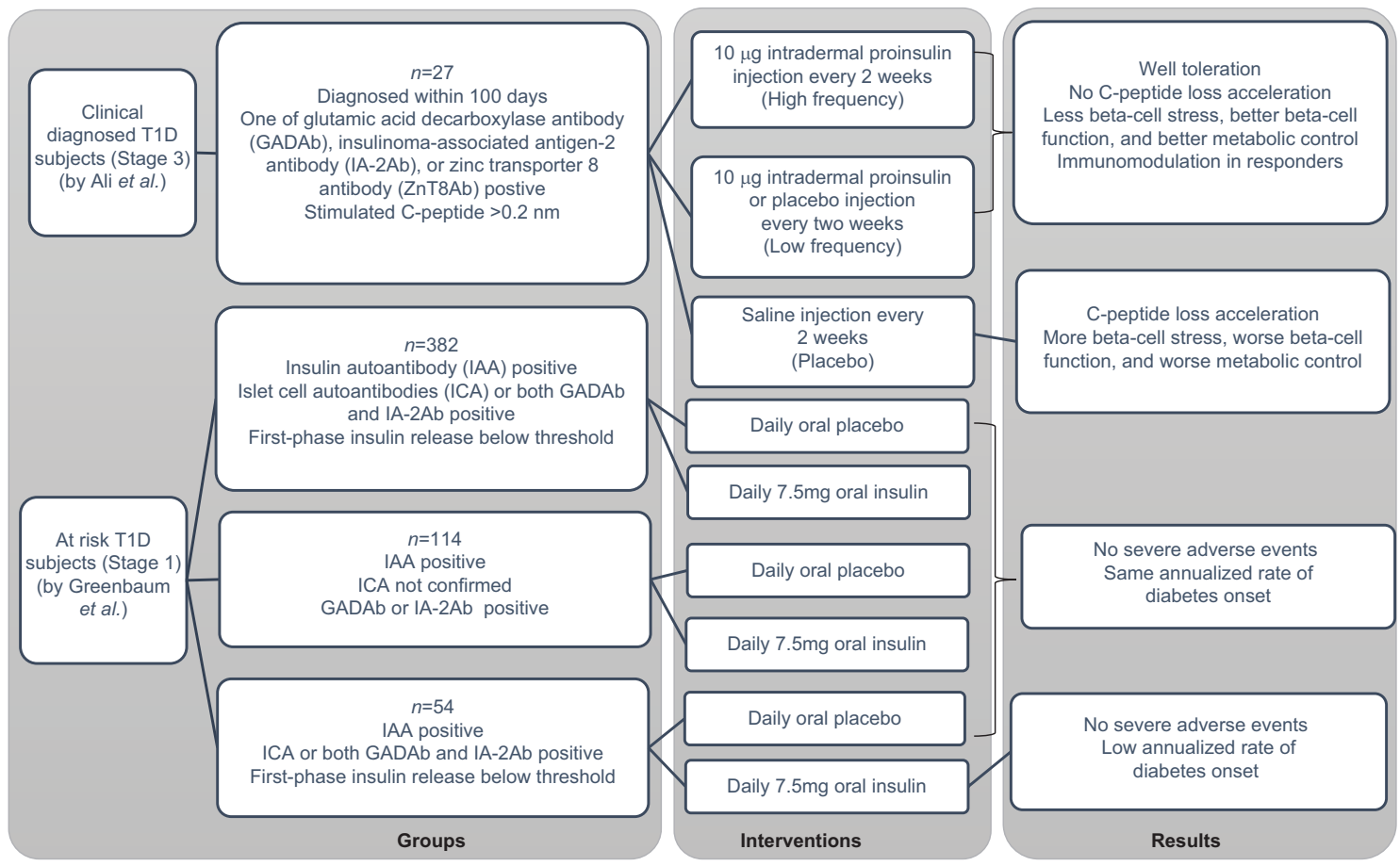

Figure 1: Two insulin immunotherapy trials. The left panel shows the recruitment design: Ali et al. recruited 27 clinically diagnosed subjects according to their criteria and Greenbaum et al. recruited subjects at high Type 1 diabetes risk and divide them into three strata according to different criteria. The middle panel shows the different treatment or placebo interventions given to each group. The right panel shows summarized results from different groups

into C-peptide responder and non-responder groups. They find that compared with the baselines and non-responders, the responder group shows a higher IL-10 level in response to proinsulin, a declining trend of interferon-gamma response at the $1^{\text {st }}$ month, higher levels of transcription factor FoxP3 expression in $\mathrm{T}_{\text {reg }}$ (especially memory $\mathrm{T}_{\text {reg }}$ ), and a lower proportion of antigen-experienced CD8 T-cells specific for beta-cells. They also report that the proinsulin/C-peptide ratio is higher compared to the baselines in non-responders but remain unchanged in responders.

Safety is the first concern when applying the ASI in human autoimmune diseases. Hypersensitivities and disease exacerbation have occurred in some ASI trials. ${ }^{[6]}$ Researchers' understanding of PIT continues to evolve. Ali et al. confirmed that intradermal proinsulin PIT is well tolerated and does not accelerate C-peptide loss in T1D subjects. The treatment groups or responders show less beta-cell stress, better beta-cell function, and better metabolic control compared to the placebo group. The previous ASI studies have suggested several mechanisms to explain immunotherapy effects in T1D. ${ }^{[6]}$ Ali et al. confirmed the treatment-induced IL-10 response and high FoxP3 expression in inflammation controlling $\mathrm{T}_{\text {reg }}$ cells. Together, their work provides a better understanding of the PIT and supports future efficacy studies.

\section{Oral Insulin Immunotherapy}

Greenbaum et al. studied the protective effects of oral insulin on T1D relatives who are defined as Stage 1 T1D with confirmed insulin autoantibodies but normal glucose tolerance. Subjects are divided into primary stratum and three secondary strata, as shown in Figure 1. Subjects receive double-masked human recombinant insulin in oral capsules or placebos. The researchers perform oral glucose tolerance tests to assess their T1D development every 6 months for 2.7 years.

Greenbaum et al. reported no severe adverse events and no hypoglycemia. They evaluate the effects of oral insulin by the elapsed time from receiving the treatment to $T 1 D$ diagnosis. As a result, the annualized rate of new T1D onset is 


\section{Cao: Insulin immunotherapy for T1D}

similar between the treatment and placebo groups except in secondary stratum 1, as shown in Figure 1. In addition, they report that subjects who adhere to the treatment have a lower annualized rate of T1D onset.

Oral insulin has inhibited T1D development and attenuated islet autoimmunity in young, pre-diabetic mice but has failed to do so in several human T1D prevention trials. ${ }^{[7]}$ Greenbaum et al. reported that oral insulin is not effective in T1D prevention generally. Interestingly, they find that the first-phase insulin release and treatment adherence may affect the results which support further studies on subjects with low first-phase insulin.

\section{Discussion}

In the future, the safety and efficacy of oral insulin are worth to be investigated in Stage 1 T1D with low first-phase insulin release, Stage 2 T1D with abnormal glucose tolerance, and newly diagnosed Stage 3 T1D individuals. Equally important, the protective effects of intradermal insulin in individuals at T1D risk could be evaluated. Genetic background, age at onset, types of first appearing diabetesrelated autoantibodies, body mass index, age at recruitment, and gender can be matched between the treatment and placebo groups. Hence, further trials can compare the efficacy of intradermal and oral insulin. The association between phenotype, genotype, and immunomodulatory effects, including induction of clonal anergy or deletion, and induction of regulatory cells or immune deviation can further be studied in subgroups with protective benefits. This may explain the difference between responder and non-responder groups to the treatments.

\section{Conclusion}

Injections of human proinsulin peptides have immunomodulation effect which maintains or improves beta-cell functions in T1D patients. Oral insulin intake does not change the annualized rate of diabetes onset in individuals at the early T1D stage.

\section{References}

1. Ali MA, Liu Y-F, Arif S, Tatovic D, Shariff $H$, Gibson VB, et al. Metabolic and immune effects of immunotherapy with proinsulin peptide in human new-onset Type 1 diabetes. Sci Transl Med 2017;9:eaaf7779.

2. Greenbaum C, Atkinson M, Baidal D, Battaglia M, Bingley $\mathrm{P}$, Bosi $\mathrm{E}$, et al. Effect of oral insulin on prevention of diabetes in relatives of patients with Type 1 diabetes a randomized clinical trial. JAMA 2017;318:1891-902.

3. Schoenborn JR, Wilson CB. Regulation of interferongamma during innate and adaptive immune responses. Adv Immunol 2007;96:41-101.

4. Larche M, Wraith DC. Peptide-based therapeutic vaccines for allergic and autoimmune diseases. Nat Med 2005;11:S69-76.

5. Bonifacio E, Ziegler A-G, Klingensmith G, Schober E, Bingley PJ, Rottenkolber $\mathrm{M}$, et al. Effects of high-dose oral insulin on immune responses in children at high risk for Type 1 diabetes the pre POINT randomized clinical trial. JAMA 2015;313:1541-9.

6. Anderton SM. Peptide-based immunotherapy of autoimmunity: A path of puzzles, paradoxes and possibilities. Immunology 2001;104:367-76.

7. Skyler JS, Krischer JP, Wolfsdorf J, Cowie C, Palmer JP, Greenbaum C, et al. Effects of oral insulin in relatives of patients with Type 1 diabetes: The diabetes prevention trial Type 1. Diabetes Care 2005;28:1068-76. 